\section{Effect on the Contraction of the Clot}

In the first experiment, with impure penicillin, it was noticed that in the tube containing 6,250 units per $\mathrm{ml}$., in which clotting was much delayed, the clot did not contract. In the observation with pure penicillin the whole of the serum was drawn off after 24 hours and its volume measured. The amount of serum obtained from the control was considerably greater than that from any of the tubes containing penicillin, indicating that even so little as 340 units per $\mathrm{ml}$. had some retarding effect on the contraction of the clot.

\section{Conclusions}

These results have no bearing on the systemic application of penicillin, as the concentration of the drug in the blood is then in the region of only 1 unit per $\mathrm{ml}$. or less. However, it is quite easy to introduce locally sufficient to interfere seriously with coagulation and contraction of the clot.

It would appear, therefore, that the local use of penicillin in an operation where the formation of a firm blood clot is of importance should be limited to washing out the cavity with a solution of the drug not exceeding 100 units per $\mathrm{ml}$. This could, of course, be combined with intramuscular injections if there is any reason to fear wound infection by penicillin-sensitive organisms.

\section{IRRADIATION OF GASTRIC CANCER*} BY

G. CRANSTON FAIRCHILD, M.R.C.S., D.M.R.E. Late Morris Research Fellow and Radiotherapist, Mount Vernon Hospital, Northwood

AND

\author{
ALAN SHORTER, M.B., B.S., F.R.C.S. \\ Surgeon to E.M.S., Mount Vernon Hospital, Northwood
}

While encouraging progress has been made in dealing with cancers in more superficial and accessible sites, cancer of the stomach still remains a problem. It is responsible for 13,000 deaths annually in Great Britain, and 40,000 in the U.S.A. (Wilbur and Shenson, 1942)-that is, 33\% of all cancer deaths in America (Mullen, 1941). But, worse still, its highest incidence in men and women is between 40 and 60 years, when they have established a place in life, a home, and a reputation ; when they are most useful to their profession and their country, and most necessary to their families (Ogilvie, 1945). It is the commonest type of cancer, with the worst results from treatment, for on the average only $1-4 \%$ of all cases are alive after five years following curative surgery, and only about four months' prolongation of life can be obtained by palliative operations (Livingston and Pack, 1941).

What can be done to decrease this annual toll of valuable lives? Apart from the possibility of prevention, about which we are able to do little until we know more of the causal factors, the problem is one of diagnosis and treatment. Early diagnosis is particularly important, for we know the vast majority of patients can be cured if treated by radical surgery when the disease is localized to the stomach. But at present some $80 \%$ of cases of gastric

*A paper read at a symposium on gastric cancer at a combined meeting of the Radiological Section of the Royal Society of Medicine, the Faculty of Radiologists, and the British Institute of Radiology on Dec 14, 1946. cancer are beyond any hope of cure by surgery alone when first brought to the surgeon (Ogilvie, 1938). In spite of the tremendous advances that have been made in the surgical treatment of this disease during recent years, radical surgery can hardly become more radical, for physiological or technical reasons. Its limitations are set by Mayo's dictum that a live patient is one of the essentials of a successful operation, and not by timidity and lack of enterprise by surgeons (Ogilvie, 1945). But what of irradiation-for post-mortem findings show that over $20 \%$ of people dying of gastric cancer have the disease still confined to the stomach and immediately adjacent lymph glands (Livingston and Pack, 1939)? Owing to the impossibility of a surgical cure in such a vast number of cases, many other forms of treatment, including irradiation, have been tried in the past, but without striking success. Hardly had Roentgen discovered $x$ rays in 1896 when Despeignes applied them to the external irradiation of a gastric cancer.

There is no need for us to recall the different forms and combinations of irradiation applied to this viscus from then to the present day. It should suffice merely to make some general remarks on their limitations and shortcomings.

External irradiation fails because of the deep situation of the gastric tumour and its close relation to other vital organs-both factors tending to prevent adequate tumour dosage-and because of the high but variable radioresistance of gastric cancer cells, possibly increased by infection. This high resistance may be only apparent, owing to limitation of the tumour dose by severe general and skin reactions. Movements of the tumour make accurate centring of the $x$-ray beam at repeated exposures difficult to obtain. Livingston and Pack (1941) stated that most patients will not survive long enough to benefit from prolonged external irradiation alone because of the profound state of malnutrition many of them attain.

Contact therapy with the Chaoul tube covers a relatively small area $(2 \mathrm{~cm}$. diameter) in each field with very little penetration at low voltage. Pack (1939) found that only $22 \%$ of the surface dose penetrated to $1 \mathrm{~cm}$. below the surface. For comparison, Fairchild finds that $32 \%$ of the surface dose of high-intensity irradiation penetrates to $10 \mathrm{~cm}$. below the surface with the technique he has been using. With the Chaoul tube bulky tumours could not be destroyed by single surface doses at one operation, but needed repeated exposures. This could be done only through some kind of fistula or pouch formation. With such a small field of $2 \mathrm{~cm}$., repeated applications would be required for most inoperable tumours, with the obvious difficulty of adequate and uniform dosage. Also, such localized irradiation would have no appreciable effect on any glandular or other local spread, the presence of which is the usual reason for inoperability.

Radon seed implantation has the same difficulty of uniform and adequate dosage (as shown by subsequent radiography) when introduced at operation from either serous or mucous surface, or through a gastrostomy opening or the oesophagoscope. There are also dangers of haemorrhage and infection and perhaps perforation due to massive necrosis of the tumour, while the adjacent field of spread does not receive adequate irradiation.

Intracavity irradiation with the five-way tube described by Livingston and Pack (1941) has, they claim, the advantages of easy application, fractionation of doses, avoidance of damage to adjacent vital organs, freedom from dangers of haemorrhage, infection, and perforation, and no interference with food intake. But they had not at that time determined how effective a total dose patients would stand, the most effective fractions into which to divide the total dose, or the results as regards palliation or cure. Used 
alone it would appear not to have much effect on local spread, but combined with direct irradiation it might perhaps be used to supplement the dose reaching the centre of large gastric tumours.

Many people think that irradiation not only shortens the patient's life but also adds to his sufferings, and therefore is quite unjustifiable. While this may be true of many of the other methods of treatment we maintain that direct irradiation at high intensity has none of these disadvantages.

\section{Direct Irradiation}

Because of the lack of any striking results from these forms of attack on gastric cancer, Fairchild (1935) first conceived the idea of direct irradiation of deep-seated tumours exposed temporarily at operation. Owing to difficulties associated with anaesthesia and the length of time required to give an adequate dose (at the rate of $40 r$ per minute - the maximum possible at that time) a compromise was attempted. Some cases of rectal cancer were treated by external irradiation applied posteriorly, after turning back skin-flaps over the sacrum and coccyx. By this means adequate tumour dosage was not obtained, so fractional doses were applied daily for a week or ten days. But the wounds became infected when kept open for so long, and the method was abandoned. Further progress was held up until 1944, when Fairchild and Shorter began to work out a technique for the direct irradiation of gastric cancers exposed temporarily at operation as had been originally intended, instead of merely turning back skinflaps. Using the intensities then available (700 $r$ to $2,000 r$ per minute), the method was later elaborated to deal with cancers of the oesophagus, pancreas, colon, etc.

\section{Advantages of the Method}

1. More accurate information about the size, shape, position, and extent of the primary lesion, and any local or general spread, can be obtained at the exploratory operation than can be found out by any other methods, either clinical or laboratory. In the thorax, where we can usually make a fairly satisfactory radiological examination, the extent of a cancer is almost invariably greater than it would seem from $x$-ray films. So in the abdomen the growth is nearly always more extensive than can be determined by the usual examinations and investigations. According to Walters et al. (1942), exploratory operation is the only reliable way of deciding the correct form of treatment.

2. Biopsy specimens can usually be obtained to help confirm the diagnosis. With palpable glandular involvement this can be done safely, but incision of the tumour may spread tumour cells or lead to perforation owing to subsequent massive irradiation. Thus biopsy may not be practicable in all cases, but being able to see and feel the tumour should make the diagnosis more certain than when relying on external methods of examination alone.

3. A more accurate and effective dose of irradiation at high intensity can be given direct to the tumour and field of local spread without irradiating such a large volume of normal tissue, including vital organs and skin, unavoidable when irradiating from the skin surface. This may be of great importance in an already anaemic and cachectic patient.

4. More accurate centring of the $x$-ray beam is possible under direct vision than when relying on $x$-ray films or clinical examination alone.

5. The skin is left practically intact, and will permit further full dosage to be given externally at a later date should this be deemed necessary. Supplementary external irradiation was given later in some of our earlier cases, beginning about ten days after operation, as we felt that the initial dose was too small to cure. Some had further external irradiation after some months.

6. Various operations to relieve obstruction can be performed before the irradiation, at the same or an earlier and less exten- sive laparotomy, such as gastrostomy for cardiac obstruction and gastro-jejunostomy for pyloric lesions. Though the obstruction may not be severe it increases owing to fibrosis of the tumour after irradiation. In hopeless cases these operations can be done without irradiation to relieve symptoms

\section{Preliminary Investigations and Pre-operative Preparation}

Diagnosis and localization of the tumour are achieved as accurately as possible by clinical, radiological, gastroscopic, oesophagoscopic, and laboratory investigations. The general condition of the patient is investigated as regards possibility of metastases, state of nutrition, and fitness for anaesthesia and operation.

Patients with cancer of the upper alimentary tract are often bad risks for operation or irradiation. This varies considerably with the site and type of growth, the degree of interference with nutrition, infection of the tumour or even the whole mucosa, and toxaemia from the growth. The patient with cancer at the cardia or pylorus is usually a worse risk than the patient with a mid-gastric lesion, owing to the more profound disturbance of nutrition, even amounting to gross deficiencies of many vitally important substances. These deficiencies must be made good, before any extensive operation or intensive irradiation is carried out, by means of a high-protein, high-carbohydrate, low-fat diet, with extra fluids, iron, salts, and vitamins when necessary (Payne, 1940 ; Reid, 1941 ; Garlock, 1942 ; Ravdin et al., 1943; Wangensteen, 1943). Infection is combated in the tumour, or in the stomach generally in pyloric obstruction, by daily gastric aspiration and lavage with weak hydrochloric acid solution. Dental sepsis, so often associated with upper tract cancers, is attended to before general anaesthesia. Routine coughing and breathing exercises are begun before operation and continued as soon as possible after operation, to maintain a clear airway and good expansion of the lungs (Shorter, 1944). This is the most important measure in avoiding lung complications after upper abdominal operations, especially when the thorax is opened as well.

\section{Surgical Approaches}

Owing to the tendency of the growth to spread along the stomach, or to lymphatics above, behind, or below the stomach, the exposure must be such as to enable the direct irradiation to be carried out in an antero-posterior plane ; otherwise uniform irradiation of the tumour area would not be possible. It follows that the left transthoracic approach is unsuitable for direct irradiation even for the cardiac area and lower oesophagus (Shorter, 1946).

This may be in one or two stages (Fairchild and Shorter, 1945). (1) When there is well-marked cardiac or pyloric obstruction gastrostomy, gastrojejunostomy, or even jejunostomy may be necessary before the patient's condition can be improved sufficiently to stand the wider exposure and irradiation. This is usually done through a small upper left paramedian incision, making exploration of the tumour and the whole abdomen possible. Many cases have in this way been found unsuitable for irradiation owing to unsuspected widespread metastases. (2) When the patient's condition seems satisfactory the abdomen is explored through a different incision more suitable for extension, even into the thorax if necessary. We have usually followed this practice when the condition of the patient will allow it, so as to avoid the two operations advocated by Garlock (1941, 1942). Also it saves moving the patient between making separate incisions for the exploration and the main exposure (Churchill and Sweet, 1942a, 1942b). The form of treatment to be carried out cannot be decided upon beforehand, 
so preparation must be made for all contingencies so far as is possible. The abdomen must be thoroughly explored before making an extensive exposure, especially before opening the thorax. It would seem to be unwise, and unfair to the patient, to open his thorax and divide his diaphragm before estimating carefully the extent of spread in the abdomen (Shorter, 1946).

(a) Midline Incision.-In our early cases the main approach was through a midline incision from the sternum, excising the xiphoid, to the umbilicus or beyond. With a narrow subcostal angle, the wound was widened by dividing each costal margin and forcibly retracting. Then the tumour was mobilized to bring it down into the open wound. This method was soon abandoned in favour of leaving the tumour in situ and retracting the abdominal and perhaps thoracic wall to give direct and wider exposure.

(b) Angular Abdomino-thoracic Incision.-For upper gastric tumours a transverse incision was made through the sixth and seventh left costal cartilages from the upper end of the midline incision, and continued round in the fifth space to the posterior axillary line. The thorax was opened and the left side of the diaphragm divided down to the oesophageal hiatus, the incision skirting round the left edge of the pericardium. This method caused less operative shock and decreased the risk of spreading the disease by lymphatic embolism, owing to decreased manipulation of the tumour. Also it gave better access to the cardiac region, though some difficulty was experienced in retracting the angular flap containing the divided left costal margin.

(c) Oblique Abdomino-thonacic Incision.-In our later cases this difficulty was overcome by an oblique incision across the upper abdomen, from the ninth right to the seventh left costal cartilage, in line with the outer part of the fifth left interspace. The incision was continued through the left costal margin lower down into the fifth interspace, the thorax opened and the diaphragm divided as before. This gave a wide straight opening with much better direct access to the whole stomach.

We have found this oblique incision most useful for gastrectomy and oesophago-gastrectomy. Garlock (1946) has recently described a somewhat similar combined abdomino-thoracic approach to the lower oesophagus and cardia, but he explores the abdomen through an upper left pararectal incision, which may be continued into the thorax through the ninth left interspace.

\section{Points in the Operative Procedure}

When operations to relieve obstruction are necessary they are done before the irradiation, and when possible in an area of the stomach not to be irradiated. Irradiation of normal viscera, so far as is possible, is avoided by displacement and packing out of the field. In our earlier cases lead screening was used to protect the skin edges of the wound. More recently we have introduced a special sterilized applicator into the wound. Sterilized oiled silk protects the field against infection during irradiation but allows accurate visual centring of the tumour in the $x$-ray beam. To avoid shock produced by frequent moving, a special trolley has been designed on which the patient can have both the operation and the irradiation.

\section{Radiological Data}

The apparatus consists of two Metropolitan Vickers constantly evacuated $x$-ray tubes of the $250-\mathrm{kV}$ type. Special modifications in the layout and in various components of this apparatus have been made, so that both tubes may be used simultaneously in treating a single lesion. This has been done to increase the intensity above that available with the standard apparatus. The lower tube has a vertical traverse only, whereas the overhead tube has a vertical and horizontal traverse. The beam of each tube can be rotated through 300 degrees in a vertical plane. With the use of radiation giving a half-absorption value of $1.7 \mathrm{~mm}$. $\mathrm{Cu}$, the available surface intensity is of the order of 1,000 $r$ per minute from each tube. In each case the focal skin distance is $21.7 \mathrm{~cm}$. and the field $13 \mathrm{~cm}$. in diameter.

Methods of Irradiation.-In our original method only one tube was used to administer a single dose of radiation direct to the lesion. This was followed in ten days by a further dose given through the skin. In our later cases this method was modified in an attempt to give an adequate total dose of irradiation at one exposure, with more uniform distribution, so as to avoid the necessity for subsequent external irradiation. For this purpose the overhead tube was used as before, while the lower tube, with the $x$-ray beam directed upwards, was arranged beneath the patient, with the canvas trolley-top alone intervening. The central ray of each beam was so directed as to pass through the centre of the lesion. In our first method, with one tube, the total initial tumour-surface dose was $500 r-1,200 r$, the latter figure being chosen as a maximum because it had been effective in certain superficial lesions. Also it was thought that a larger dose might cause massive necrosis of tumour tissue, with a risk of haemorrhage and perforation, there being no precedent to indicate the probable effects of such high tumour doses given with a maximum of two to five minutes. In our second method a provisional average tumour dose of $1,300 r$ was decided on as a minimum throughout the tumour, entailing a surface dose of $1,300 r$ from the under-couch tube and 1,600 $r$ direct to the lesion from above. This latter dose is being gradually increased.

Post-operative and Irradiation Reactions.-Immediate reactions have been no more severe than would have been expected after the operation alone, which often involved extensive exposure of the viscera, with both thorax and abdomen widely open together, and much manipulation during isolation of the tumour. Even after giving 3,000 $r$ in three minutes there has been remarkably little irradiation reaction, which has come on seven to ten days later, when the operation reaction has passed off. It has been mostly limited to nausea and anorexia, with rarely any vomiting or diarrhoea. Blood changes have been less severe than usual after external irradiation, when the dose is spread over a longer time and a far greater volume of tissue and blood is treated. Irradiation reaction has been more severe and the recovery much slower after the external irradiation given to certain of our earlier cases some months after the operation and direct irradiation, even though the patients were generally more fit for the later treatment, which was a relatively smaller dose spread over a few weeks.

\section{Results of Treatment}

During the past two and three-quarter years 32 cases of inoperable gastric cancer have been investigated. Of these patients three refused all treatment and left hospital, and one was considered unfit for exploratory operation, being a man of 80 with a large palpable tumour of the lesser curve. The remaining 28 cases were explored. Three of these were unfit for further treatment-one due to widespread growth and poor general condition; another due to extensive residual growth and insuperable adhesions following partial gastrectomy; and the third due to extensive growth with perforation of a carcinomatous ulcer. Ten of those explored were found unfit for direct irradiation owing to too extensive growth or poor general condition, or both, and had palliative operations only.

This leaves 15 cases that were treated by direct irradiation. These can be divided into two groups-first, a group of seven cases in which the irradiation was purely palliative owing to obvious spread beyond the possible field of irradiation or to poor general condition. Of these, one is 
alive and well after five weeks; one lived six months, one five months, and two four months; while the rest died sooner. In the second group of eight cases the growth appeared to be limited to an area that could be irradiated. Of these, one lived 24 months, two 15 months, one seven months, one four months, and one three months after treatment. The remaining two died within the first weekone from bilateral pulmonary collapse while the wound was being closed, the other from spontaneous pneumothorax on the opposite side four days after operation.

\section{Details of Cases}

The following are brief details of some of our cases in chronological order, as we proceeded with and modified our technique.

Case 1.-A man aged 35, with carcinoma of the lesser curve, had a laparotomy on Feb. 15, 1944, via the upper midline incision, widened by division of both lower costal margins. The tumour was a large elongated flat plaque extending from $2.5 \mathrm{~cm}$. short of the pylorus to the cardia ; its surface was stony-hard, irregular, and with subserous spread. It measured $13 \mathrm{~cm}$. vertically, $7 \mathrm{~cm}$. antero-posteriorly, and $4.5 \mathrm{~cm}$. thick. The rest of the stomach was normal. Spread to glands or viscera was not found. A biopsy was not made, but surgeon, radiotherapist, and pathologist were all sure of the diagnosis. The tumour was irradiated with one tube, the maximal tumour dose being $1,000 r$ in one minute. External irradiation was begun nine days later, amounting to a maximal tumour dose of $970 r$ in 12 days. The total maximal tumour dose was $1,970 r$ in 21 days. The tumour decreased very much in size, with marked contraction of the stomach also. The patient's appetite soon returned, and he was able to eat any food without indigestion. As his stomach became smaller it emptied more rapidly, so that he had to take small meals frequently. He gained over 2 stone $(12.7 \mathrm{~kg}$.) in weight after treatment, and returned to work as a newspaper reporter within six months. He was readmitted to hospital at the end of December, 1945, and died from widespread metastases in February, 1946, just over two years after the direct irradiation. Post-mortem examination showed growth in the whole stomach, throughout the chest and abdomen, and in the brain. This was our first patient; he had a relatively small dose of irradiation and lived two years, most of which time he was free from any symptoms.

Case 2.-A man aged 67, with carcinoma of the pyloric antrum, had a laparotomy on Feb. 22, 1944, via the upper midline incision. The tumour was a hard irregular cylindrical mass encircling the pylorus and pyloric antrum, $6.5 \mathrm{~cm}$. long and $5 \mathrm{~cm}$. across. Glands in the lesser curve were taken for biopsy, and were reported on as early infiltration with carcinoma cells compatible with stomach primary carcinoma. The tumour and glands were irradiated with one tube, the maximal tumour dose being $1,000 r$ in 1.5 minutes. External irradiation was begun in nine days, but was stopped after a few days (275 $r$ maximal tumour dose) owing to the development of a lung abscess. External irradiation was restarted six weeks later with a further $1,089 r$. The total maximal tumour dose was $2,364 r$ in 56 days. Almost complete pyloric obstruction developed owing to fibrosis and contraction in tumour after irradiation. No improvement took place by May.30,1944, so a gastro-jejunostomy was performed. The patient died of heart failure within a week. The second operation could have been avoided had a short-circuit been done at the first operation, though the patient was in poor condition then. The irradiation given was inadequate, but a larger dose was precluded by the patient's poor condition.

Case 3.-A man aged 60 , with carcinoma of the greater curve, had a laparotomy on April 4, 1944, via the upper midline incision. The tumour was a large irregular stony-hard mass on the greater curve in the pyloric antrum measuring $13 \mathrm{~cm}$. transversely, $7 \mathrm{~cm}$. vertically, and $7.5 \mathrm{~cm}$. antero-posteriorly, with an ulcer $7.5 \mathrm{~cm}$. across facing into the lumen of the stomach. The tumour was attached to and invading the pancreas. There was a subserous spread on the surface of the tumour, and some was removed for biopsy. The report stated that there was a large spheroidal and round-celled adenocarcinoma of the stomach with well-marked mucoid change. No other spread was found. The tumour was irradiated with one tube-maximal tumour dose, 1,000 $r$ in 1.5 minutes. External irradiation was begun nine days later, amounting to a maximal tumour dose of $991 r$ in six days. The total maximal tumour dose was $1,991 r$ in 16 days. His pain disappeared within a few weeks of the direct irradiation, his general condition quickly improved, his appetite soon returned, and he returned to work as a stoker five months after direct irradiation, having gained $1 \frac{1}{2}$ stone $(9.5 \mathrm{~kg}$.) in weight. He was readmitted for further external irradiation on Nov. 23, 1944, as it was thought the initial dose was too small to cure the lesion. The second maximal tumour duse was 2,646 $r$ in eight days. Systemic reaction was more severe and his recovery was much slower after this, but he continued to improve. He began vomiting at the end of March, 1945 , owing to obstruction at gastro-jejunostomy by contraction of the growth after treatment. He continued to vomit but refused further operation until the end of June, 1945, by which time his general condition was very poor. At this operation the duodenum proximal to the anastomosis was joined to the jejunum beyond, but he died a few days later from intestinal obstruction by a volvulus after the operation. Post-mortem examination showed growth in the stomach, mostly outside the treated area. He lived 15 months after the direct irradiation, and might well have lived longer if he had allowed us to perform the second operation sooner.

Case 4.-A woman aged 54, with a leather-bottle stomach from pylorus to cardia, had a laparotomy on May 9, 1944, via the upper midline incision. The stomach was stony-hard from pylorus to cardia. Glands were found high in the lesser curve and below the pylorus. Biopsy of the gland showed undifferentiated glandular carcinoma. The tumour and glands were irradiated with difficulty with one tube, as it was impossible to get the whole lesion into the field of irradiation. The maximal tumour dose was $950 r$ in 1.6 minutes. External irradiation was begun nine days later, amounting to a maximal tumour dose of $2,310 r$ in 13 days. The total maximal tumour dose was 3,260 $r$ in 23 days. She improved for a time and was quite free from pain. Her appetite did not improve, and she died at home four months later. A necropsy was not done.

Case 5.-A woman aged 57, with carcinoma of the abdominal oesophagus involving the cardia, had an angular abdominothoracic incision on May 16,1944. The abdomen was explored and a small stony-hard pear-shaped tumour $5 \mathrm{~cm}$. long by $2.5 \mathrm{~cm}$. across was found encircling the abdominal oesophagus. There were no signs of spread. The incision continued into the thorax, but no spread was found there. Operation was not possible owing to the poor general condition. The tumour was irradiated with one tube, with a maximal tumour dose of $1,000 r$ in 4.26 minutes. External irradiation was begun in nine days, amounting to a maximal tumour dose of 2,541 $r$ in 11 days. The total maximal tumour dose was 3,541 $r$ in 20 days. Her general condition improved, and swallowing, which was previously limited to fluids, was very much better. She gained some weight. Further external irradiation to cover the length of the mediastinum and lesser omentum was begun on March 12, 1945. The maximal tumour dose was $2,046 r$ in four days. The systemic reaction was severe, but her general condition slowly improved. Swallowing became almost normal. Then she developed a pyrexia and died suddenly on Aug. 23, 1945-15 months after the direct irradiation. Necropsy showed death to be due to broncho-pneumonia and cirrhosis of the liver. There was no sign of viable cancer cells in the irradiated area, but some were found in the right lung base. This was the first gastric case for which we opened the thorax and abdomen together.

Case 6.-A woman aged 42, with carcinoma of the cardia, had an angular abdomino-thoracic incision and her abdomen explored on Nov. 14, 1944. A tumour about $6 \mathrm{~cm}$. in diameter was found involving the cardia and abdominal oesophagus. Glands were found around the left gastric vessels; no other sign of spread was discovered. Biopsy of glands did not show any involvement with carcinoma cells. Incision continued into the thorax, but the general condition of the patient was too poor to allow direct irradiation or gastrectomy to be carried out, and the wound had to be closed hurriedly. Another attempt was made to expose the tumour for irradiation on Jan. 16, 1945, through the oblique abdomino-thoracic incision. 
Great difficulty was experienced with adhesions, and again the operation had to be terminated rapidly before completion. The patient died some 36 hours later. Post-mortem examination confirmed the carcinoma encircling the cardia and lower abdominal oesophagus, causing complete obstruction. No cther possible signs of spread were observed except the glands found at operation. Unfortunately this patient had been treated as a case of achalasia for some eight months, in spite of losing 2 stone $(12.7 \mathrm{~kg}$.) in weight and having gradually progressive symptoms.

Case 7.-A woman aged 46, with carcinoma of lesser curve, had a laparotomy on Nov. 21, 1944, via the upper midline incision. A saddle-shaped tumour $8 \mathrm{~cm}$. transversely by $5 \mathrm{~cm}$. vertically by $4 \mathrm{~cm}$. thick was present on the lesser curve in the pyloric antrum and body. Hard glands were found along the lesser curve and below the pylorus and body of the pancreas. Biopsy of glands revealed infiltration by mucus-secreting carcinoma, showing little differentiation and increased fibrous stroma as in leather-bottle stomach. The tumour and glands were irradiated by two tubes, the maximal tumour dose being 1,528 r. in 2.4 minutes. The patient withstood operation and irradiation very well, but suddenly collapsed and died just as the wound was being closed. Necropsy showed complete collapse of both lungs, for which no cause, either intra- or extrapulmonary, was found.

Case 8.-This patient, a woman aged 54, had a carcinoma of the lesser curve. Her abdomen was explored via an oblique abdomino-thoracic incision on March 23, 1945, and a saddleshaped tumour measuring $8 \mathrm{~cm}$. transversely by $4 \mathrm{~cm}$. vertically by $4 \mathrm{~cm}$. antero-posteriorly was found high on the lesser curve, extending a quarter-way round the lumen on each surface; it was stony-hard, nodular, with subserous spread. There were glands along the lesser curve. Biopsy showed moderately differentiated adenocarcinoma with much fibrous stroma invading the lymph gland. There was no other sign of spread. The incision was continued into the thorax. The tumour and glands were irradiated with two tubes, a maximal tumour dose of $1,542 r$ being given in three minutes. Her general condition improved, her appetite returned, and she gained in weight and lost all dyspepsia. Her stomach had contracted considerably some five months after irradiation, and she had to have frequent small meals. Later, she would not take much food because of the return of pain, and then vomiting began. Her abdomen was explored through a left paramedian incision on Sept. 28, 1945, when much clear fluid was found, with miliary peritoneal spread of carcinoma. A palliative jejunostomy was performed, but she died within a week. Necropsy showed widespread involvement of the parietal and visceral peritoneum and omentum, with metastases. The stomach was greatly contracted and showed infiltration by growth from pylorus to cardia.

Case 9.-A man aged 68, with carcinoma of cardia, had the abdomen explored through an oblique abdomino-thoracic incision on Feb. 13, 1945. A tumour $6 \mathrm{~cm}$. transversely by $4 \mathrm{~cm}$. vertically by $3.5 \mathrm{~cm}$. antero-posteriorly was found at the cardia. There were glands in the lesser curve and above the cardia, but no spread was observed elsewhere in the abdomen. Biopsy of the glands showed poorly differentiated adenocarcinoma invading the lymph-gland. The incision was continued into the thorax, but no spread was found there. The tumour and glands were irradiated with two tubes, the maximal tumour dose being $1,196 r$ in 1.8 minutes-smaller than intended because the lower tube became unstable and was turned off. The patient progressed very well for four days, when for no apparent reason spontaneous pneumothorax suddenly developed on the right side, causing rapid death from heart failure in spite of all attempts at resuscitation.

\section{Conclusions}

To summarize our results, 15 out of 32 cases were treated by direct irradiation. In eight of these the disease appeared to be limited to an area that could be irradiated, and of this group one lived 24 months, two 15 months, one seven months, one four months, and one three months after treatment.

Although the series is small we feel sure that direct irradiation at high intensity will prove to be a rational and hopeful advance in the treatment of cancer of the stomach and other viscera. Whether the cancer is rendered inoperable by the poor general condition of the patient or by irremovable local spread, this method will often succeed where surgery alone must inevitably fail. Though wider exposure is needed than for surgery alone, with adequate precautions the whole procedure should not be so shockproducing as is wide surgical excision.

Exploratory operation is the only reliable way of deciding the correct form of treatment, and the hospital of the future will have a radiotherapy room adjoining the operating theatre to facilitate direct irradiation.

It is more reasonable to irradiate most what most needs irradiating-i.e., the tumour and field of local spread-and not the overlying skin or intervening vital tissues.

Biopsy may not be practicable in all cases, as it is not altogether devoid of risk when there is no obvious glandular spread. But even biopsy is not always reliable, depending as it does on choice of the right piece of tissue, and being vitiated in some cases by variations in cellular differentiation in different parts of a tumour. Being able to see and feel the tumour should make the diagnosis more certain than when relying on external methods of examination alone, and biopsy will often increase that certainty.

Of course a method involving such extensive surgical procedures and such intensive irradiation must inevitably present new problems. Owing to difficulty in obtaining a sufficient number of suitable cases we have scarcely advanced beyond the "growing-pains." There are many modifications and improvements in technique we would like to try out. We hope that we have shown that our method is a reasonable and practicable possibility, and that eventually we may be able to improve on the present appallingly low cure rate of gastric cancer.

Unless and until we can achieve earlier diagnosis and bring a far greater number of operable cases to the gastric surgeon, there will remain a vast number of victims of this dreadful disease destined to die without even the hope of palliation or amelioration of their sufferings. Much more must be done for these patients, who greatly exceed numerically the relative few with operable lesions under the present conditions of diagnosis. Only too often our attitude is one of defeatism and despair towards these people, whose needs are often desperate. Even limited palliation for relatively large numbers might well balance the success achieved by spectacular and dangerous operations only possible for relatively few (Livingston and Pack, 1941).

\section{REFERENCES}

Churchill, E. D., and Sweet, R. H. (1942a). Ann. Surg., 115, 897. (1942b). Ibid., 116, 566 .

Fairchild, G. C. (1935). British Empire Cancer Campaign Annual Report, p. 84

and Shorter, Alan (1944). Ibid., p. 40.

- and Shorter, Alan (1944),

Garlock, J. H. (1941). Surg. Gynec. Obstet., 73, 244.

- (1942). Ibid., 74, 555.

(1946). Ibid., 83, 737.

Livingston, E. M., and Pack, G. T. (1939). Results of Treatment of Gastric Cancer. Hoeber, New York.

- (1941). Amer. J. Surg., 51, 453.

Mullen, T. F. (1941). Surg. Gynec. Obstet., 72, 298.

Ogilvie, Sir Heneage (1938). Lancet, 2, 235.

(1945). Lecture on Cancer of Stomach and Oesophagus, Mount Vernon Hospital. (Unpublished.)

Payne, R. T. (1940). Brit. J. Surg., 27, 740.

Ravdin, I. S., Royster, H. P., Riegel, C., and Rhoads, J. E. (1943). Arch. Surg., 46, 871 .

Reid, M. R. (1941). J. nat. Cancer Inst., 1, 523.

Shorter, Aian (1944). Lancet, 1, 243.

(1946). Proc. royal Soc. Med., 39, 420.

Walters, W., Gray, H. K., Priestley, J. T., and Counsellor, V. S. (1942). "Proc. Mayo Clin., 17, 420.

Wangensteen, O. H. (1943). Arch. Surg., 46, 879.

Wilbur, D. L., and Shenson, B. (1942). Amer. J. Surg., 56, 94. 